\title{
Examining the presence and nature of delusions in Alzheimer's disease and frontotemporal dementia syndromes
}

\author{
Fiona Kumfor ${ }^{1,2}$ (1) | Cheng Tao Liang ${ }^{1,2}$ | Jessica L. Hazelton ${ }^{1,2}$ | \\ Cristian E. Leyton $^{2,3}$ | Cassandra Kaizik ${ }^{2,4}$ | Emma Devenney ${ }^{2,5}$ | \\ Emily Connaughton $^{6}$ | Robyn Langdon ${ }^{6}$ | Eneida Mioshi ${ }^{7}$ | John B. Kwok ${ }^{2,5,8}$ | \\ Carol Dobson-Stone ${ }^{2,5,8}$ | Glenda M. Halliday, ${ }^{2,5,8}$ | Olivier Piguet ${ }^{1,2}$ | \\ John R. Hodges $^{2,5}$ | Ramon Landin-Romero ${ }^{1,2}$
}

${ }^{1}$ School of Psychology, The University of Sydney, Sydney, New South Wales, Australia

${ }^{2}$ Brain \& Mind Centre, The University of Sydney, Sydney, New South Wales, Australia

${ }^{3}$ Faculty of Health Sciences, The University of Sydney, Sydney, New South Wales, Australia

${ }^{4}$ Memory and Cognition Clinic, RPA Hospital, Sydney, Local Health District, New South Wales, Australia

${ }^{5}$ Central Clinical School, The University of Sydney, Sydney, New South Wales, Australia

${ }^{6}$ Department of Cognitive Sciences, Macquarie University, Sydney, New South Wales, Australia

${ }^{7}$ School of Health Sciences, University of East Anglia, Norwich, UK

${ }^{8}$ School of Medical Sciences, University of New South Wales, Sydney, New South Wales, Australia

\section{Correspondence}

Fiona Kumfor, Brain \& Mind Centre, The University of Sydney, 94 Mallett Street, Camperdown, Sydney, NSW 2050, Australia. Email: fiona.kumfor@sydney.edu.au

Funding information

Australian Research Council Centre of Excellence in Cognition and its Disorders Memory Program; Appenzeller Neuroscience Fellowship in Alzheimer's Disease; National Health and Medical Research Council

\begin{abstract}
Objectives: Abnormal beliefs and delusions have been reported in some people with dementia, however, the prevalence of delusions, and their neurocognitive basis has been underexplored. This study aimed to examine the presence, severity, content and neural correlates of delusions in a large, well-characterised cohort of dementia patients using a transdiagnostic, cross-sectional approach.

Methods: Four-hundred and eighty-seven people with dementia were recruited: 102 Alzheimer's disease, 136 behavioural-variant frontotemporal dementia, 154 primary progressive aphasia, 29 motor neurone disease, 46 corticobasal syndrome, 20 progressive supranuclear palsy. All patients underwent neuropsychological assessment and brain magnetic resonance imaging, and the Neuropsychiatric Inventory was conducted with an informant, by an experienced clinician.

Results: In our cohort, 48/487 patients (10.8\%) had delusions. A diagnosis of behavioural-variant frontotemporal dementia (18.4\%) and Alzheimer's disease (11.8\%) were associated with increased risk of delusions. A positive gene mutation was observed in 11/27 people with delusions. Individuals with frequent delusions performed worse on the Addenbrooke's Cognitive Examination $(p=0.035)$, particularly on the orientation/attention $(p=0.022)$ and memory $(p=0.013)$ subtests. Voxel-based morphometry analyses found that increased delusional psychopathology was associated with reduced integrity of the right middle frontal gyrus, right planum temporale and left anterior temporal pole.

Conclusion: Our results demonstrate that delusions are relatively common in dementia and uncover a unique cognitive and neural profile associated with the manifestation of delusions. Clinically, delusions may lead to delayed or misdiagnosis. Our results shed light on how to identify individuals at risk of neuropsychiatric features of dementia, a crucial first step to enable targeted symptom management.
\end{abstract}

This is an open access article under the terms of the Creative Commons Attribution-NonCommercial-NoDerivs License, which permits use and distribution in any medium, provided the original work is properly cited, the use is non-commercial and no modifications or adaptations are made.

(c) 2022 The Authors. International Journal of Geriatric Psychiatry published by John Wiley \& Sons Ltd. 


\section{KEYWORDS}

Alzheimer's disease, C9orf72, cognitive impairment, frontotemporal dementia, primary progressive aphasia, psychosis, research domain criteria (RDoC), structural imaging

\section{Key points}

- Delusions showed high prevalence, occurring in $10 \%$ of all dementia syndromes.

- Behavioural-variant frontotemporal dementia had an increased risk of delusions.

- Most patients experience polythematic delusions, often and of ranging severity, with delusions being related to more severe attention, orientation and memory deficits.

- Delusions were more common in people with strong family history/genetic mutations.

\section{1 | INTRODUCTION}

Delusions - 'fixed beliefs that are not amenable to change in light of conflicting evidence' ${ }^{1}$ - are recognised symptoms of many psychiatric disorders, and can also occur in some neurological conditions. The nature of delusions can vary from the bizarre (e.g., the belief that a stranger has removed and replaced one's organs without leaving scars) to the mundane (e.g., that one's spouse is being unfaithful) Delusions have been reported in Dementia with Lewy Bodies and Parkinson's Disease with Dementia, and are associated with later disease stage, worse cognition and reduced insight. ${ }^{2}$ However, the extent that delusions occur in other neurodegenerative syndromes is less understood. Indeed, it has been noted that when delusions are reported in people with dementia it can contribute to misdiagnosis. ${ }^{3}$ In Alzheimer's disease (AD), estimates vary with studies reporting delusions in $16 \%-70 \%$ of patients throughout the disease course. ${ }^{4}$ In frontotemporal dementia (FTD), delusions were reported in only 2 of 86 people with behavioural-variant frontotemporal dementia (bvFTD) over a 2 year period. ${ }^{5}$ The prevalence seems to be higher in patients with FTD with comorbid motor neurone disease (FTDMND). ${ }^{6}$ Conversely, in primary progressive aphasia (PPA), delusions appear to be rare. ${ }^{7}$ In motor disorders pathologically and clinically associated with FTD, namely corticobasal syndrome (CBS) and progressive supranuclear palsy (PSP), the prevalence of delusions has been rarely studied, however, the existing evidence suggests that delusions are relatively uncommon in these syndromes. ${ }^{7}$

The limited evidence demonstrates considerable heterogeneity of findings. Previous studies have been limited by a reliance on questionnaire data, relatively small sample sizes and rare simultaneous neuroimaging. Importantly, studies which focus on single diseases alone, are likely to be insufficient to explain the presence of delusional beliefs. In the psychiatric field, a transdiagnostic approach that focuses on symptoms rather than on syndromes-referred to as the Research Domain Criteria $(\mathrm{RDoC})^{8}$-has been gaining traction since the 2010s to explain complex psychiatric phenomena. RDoC offers a new framework for understanding the biological basis of symptoms, while being agnostic regarding diagnostic syndromes. ${ }^{9}$ Such an approach is well suited to studying dementia (e.g., ${ }^{10}$ ), where diagnostic certainty in life is currently largely unachievable. Furthermore, by focusing on symptoms across diagnoses, this approach has the potential to inform theoretical models and symptoms that span neurology and psychiatry, such as delusions. ${ }^{11}$ In light of these considerations, the current study had two broad aims. First, to conduct a systematic cross-sectional review of all cases who have been seen at a dementia-focused tertiary referral clinic to examine how common delusions are across a range of dementia syndromes, and investigate the nature of delusional content, and their clinical associations. Second, to explore the neural correlates associated with delusions.

\section{2 | MATERIALS AND METHODS}

\section{1 | Participants}

This retrospective, cross-sectional study included all participants who have been seen at the Frontier dementia clinic in Sydney since its inception in 2010 and were diagnosed with a primary neurodegenerative disorder and had completed the Neuropsychiatric Inventory (NPI) at their first clinical assessment. In total, 487 participants were included: 102 AD (94 typical AD, 8 posterior cortical atrophy); 136 bvFTD; 53 semantic dementia (SD) (38 left-predominant, 15 rightpredominant); 51 progressive nonfluent aphasia (PNFA); 50 logopenic progressive aphasia (LPA); 46 CBS; 29 MND (27 FTD-MND, 2 MND); 20 PSP; based on current consensus diagnostic criteria ${ }^{12-17}$ and diagnosis by a multidisciplinary team. All controls scored above 88/100 on the Addenbrooke's Cognitive Examination (ACE), either the revised (ACE-R) or the third (ACE-III) version, with ACE-III scores converted to ACE-R scores. ${ }^{18}$ In addition to a relevant diagnosis, inclusion criteria were: a relatively early stage of the disease (i.e., living in the community and able to manage basic activities of daily living); sufficient level of English proficiency and absence of another major neurological or psychological condition that could explain the presentation. Individuals who were assessed but did not have evidence of a primary neurodegenerative disorder were excluded.

\subsection{Rating of delusions and neuropsychiatric symptoms}

Presence of delusions was established with the NPI. ${ }^{19}$ Informants were interviewed by a trained clinician to determine the presence or absence of each symptom domain. If the symptom is reported as 
present, the frequency and severity of the symptoms were rated and a composite score was calculated by multiplying frequency $\times$ severity, with higher scores denoting greater psychopathology. In addition, the clinician recorded the nature of the delusional belief.

\subsection{1 | Classification of delusional themes}

Case files were reviewed by an experienced clinician to identify the content of delusions, blind to participant diagnosis. Where classification was unclear, discussion with two other experienced clinical researchers (Ramon Landin-Romero, Robyn Langdon) was undertaken until consensus was reached. The case file review included information from the NPI as well as referral and neurology letters following the clinical assessment. The content of delusions was classified according to the Research Version of the Structural Clinical Interview (SCID-RV), ${ }^{20}$ which includes 14 types of delusions: reference, persecutory, grandiose, somatic, guilt, jealous, erotomanic, religious, control, thought insertion, thought withdrawal, thought broadcasting, bizarre and other. An additional category-misidentification-was added in light of the high prevalence of this theme in Alzheimer's disease $^{21}$ and other dementia syndromes. ${ }^{7}$ For each individual the number of types of delusions were determined to enable classification into monothematic (i.e., only one type of delusion reported) and polythematic (i.e., more than one type of delusion reported).

\section{3 | Genetic and family history screening}

A family history of neurodegenerative disease was obtained, and the Goldman score was calculated. ${ }^{22}$ A score of 3.5 or 4 is indicative of sporadic disease whereas scores $<3.5$ are indicative of familial disease. Genomic DNA was available for screening for the hexanucleotide repeat expansion in $\mathrm{C} 9$ orf72 by repeat-primed polymerase chain reaction. Expansion positive C9orf72 status was determined as previously described. ${ }^{23}$ microtubule associated protein tau (MAPT) and granulin precursor (GRN) genes were screened either by Sanger sequencing of all coding exons ${ }^{24,25}$ or by wholeexome sequencing (110 bp paired reads at 100X coverage) using the Illumina platform provided by Macrogen, Korea. Processing, mapping and variant calling of raw sequence data were performed using gold-standard Genome Analysis Toolkit pipelines. ${ }^{26}$

\subsection{Cognitive and behavioural assessment}

Global cognitive function was measured with the ACE-R or ACE-III with ACE-III scores converted to ACE-R scores. ${ }^{18}$ Attention was assessed via Digit Span Forwards and the Trail Making Test Trails A. Visuospatial-constructional skills and non-verbal episodic memory were measured using the Rey Complex Figure (RCF). Aspects of language, including naming, comprehension, semantic association and word repetition were evaluated via the Sydney Language Battery (SYDBAT). ${ }^{27}$ Executive function tasks indexed working memory (Digit Span Backwards), generativity (Letter Fluency), inhibitory control (Hayling Sentence Completion Error Score) ${ }^{28}$ and taskswitching (Trail Making Test Trails B). Behavioural change was assessed using the Cambridge Behavioural Inventory-Revised informant questionnaire $(\mathrm{CBI}-\mathrm{R}){ }^{29}$

\subsection{Neuroimaging acquisition and analyses}

Participants underwent whole-brain structural magnetic resonance imaging (MRI) using a 3 T Phillips scanner. High resolution T1-images were obtained using the following protocol: $256 \times 256,200$ slices, $1 \mathrm{~mm}^{2}$ in-plane resolution, $1 \mathrm{~mm}$ slice thickness, echo time/repetition time $=2.6 / 5.8 \mathrm{~ms}$, flip angle $=8^{\circ}$. Brain scans were available for 22 people with Delusions. FMRIB Software Library voxel-based morphometry (VBM), included in the Functional Magnetic Resonance Imaging of the Brain (FMRIB) software library package http://www. fmrib.ox.ac.uk/fsl/fslvbm/index.html was used to analyse the MRI data $^{30}$ (See Supplementary Information). A voxel-wise general linear model $(G L M)^{31}$ was created to examine voxel-by-voxel negative correlations between NPI delusions composite scores and grey matter (i.e., higher scores, lower voxel integrity), while controlling for confounding variables (diagnosis, sex, age and disease severity) (frontotemporal dementia rating scale, $\mathrm{FRS}^{32}$ ) in the Delusions + participants only. Statistical significance was set at $p<0.005$ uncorrected for multiple comparisons, with a conservative cluster extent threshold of 100 voxels, to balance the risk of Type I and Type II error. ${ }^{33}$

\section{6 | Statistical analyses}

Analyses were conducted using IBM SPSS Statistics, Version 25.0. One-way analyses of variance or independent samples $t$-tests were conducted to examine demographic and outcome clinical and cognitive variables. Categorical variables (e.g., sex, Goldman score) were analysed using chi-square tests. For all analyses, statistical significance was set at $p<0.05$ and Sidak correction for multiple comparisons was applied for post hoc analyses. Associations between presence of delusions and diagnosis was assessed using odds ratios that were estimated by means of logistic regressions with weighted observations. To examine predictors of the presence of delusions, we conducted a logistic regression analysis with ACE score, disease duration and diagnostic group included as predictor variables using the stepwise (backwards) approach.

\section{7 | Data availability}

The data generated during, or analysed as part of the current study, are available from the corresponding author on reasonable request. 


\section{8 | Reporting guidelines}

The Strengthening the Reporting of Observational Studies in Epidemiology ${ }^{34}$ cross-sectional reporting guidelines were utilised in the current study.

\section{3 | RESULTS}

\subsection{Presence and nature of delusions}

Approximately $10 \%$ of the patient cohort (48/487) experienced delusions at their first clinical assessment (Table 1). Across clinical groups, $18.4 \%$ of bvFTD, $13.8 \%$ of FTD-MND and $11.8 \%$ of AD patients were reported as experiencing delusions at presentation. When we examined the frequency and severity of delusional symptoms in the 47 of 48 cases who had been reported as having delusions, and for whom relevant NPI data were available, we found over $60 \%$ experienced delusions at least often ( $n=8 ; 17 \%)$, frequently ( $n=8 ; 17 \%$ ), or very frequently ( $n=13 ; 28 \%$ ). The delusions were rated as mild in 21 participants (45.7\%), moderate in 15 (32.6\%), and marked in 10 (21.7\%) participants.

The most common delusional theme was persecutory, followed by delusions of reference and then delusions of jealousy. Nine patients showed monothematic delusions, whereas 22 reported polythematic delusions. Of interest, 1 bvFTD patient was reported to have 5 different delusional themes, and $1 A D$ patient had 6 different delusional themes. For $17 / 48$ cases, insufficient information was available for the delusional theme to be classified.

The logistic regression model (Table 2) was significant $\left(x^{2}=37.207, p<0.001\right)$. Significant predictors in the final model were, disease duration $(p=0.025)$ and bvFTD diagnosis $(p=0.018)$ with a trend for cognition (ACE Total Score $p=0.054$ ). The final model had specificity of $90.3 \%$, although sensitivity was lower (27.7\%), with the model correctly classifying 404/480 participants.

\section{2 | Subgroup analyses}

To examine potential genetic, neural and cognitive mechanisms contributing to the presence of delusions we compared individuals who experienced delusions 'often', 'frequently' or 'very frequently', referred to as the Delusions+ group. People who reported delusions 'occasionally' were not included in the subgroup analysis. This cut-off was selected in an attempt to identify a relatively homogenous group of patients where delusional symptomatology occurred sufficiently reliably for a potential neurocognitive basis to be identified, and to minimise confounds in reporting of single instances. NPI frequency and severity data for the subgroup are shown in Figure 1.

This group included 16 bvFTD, 6 AD**, 3 FTD-MND, 3 leftpredominant SD, 1 right-predominant SD, 1 LPA. A disease control group (Delusions-) was created by matching each participant with delusions with a patient without delusions from the original cohort,




TABLE 2 Logistic regression results

\begin{tabular}{lcllll} 
& $\beta(\mathrm{SE})$ & Lower & Odds ratio & Upper & $p$ \\
\hline ACE Total score & $0.01(0.01)$ & 1.00 & 1.00 & 1.00 & 0.054 \\
\hline Disease duration & $0.12(0.05)$ & 1.01 & 1.12 & 1.24 & 0.025 \\
\hline bvFTD diagnosis & $0.76(0.32)$ & 1.14 & 2.13 & 3.99 & 0.018 \\
\hline PNFA diagnosis & $-18.71(5576.27)$ & 0.00 & 0.00 & - & 0.997 \\
\hline CBS diagnosis & $-18.75(5951.70)$ & 0.00 & 0.00 & - & 0.998 \\
\hline Constant & $-2.31(0.40)$ & & & & \\
\hline
\end{tabular}

Note: $R^{2}=0.08$ (Cox \& Snell), 0.16 (Nagelkerke). Final model $x^{2}=41.505, p<0.001$.
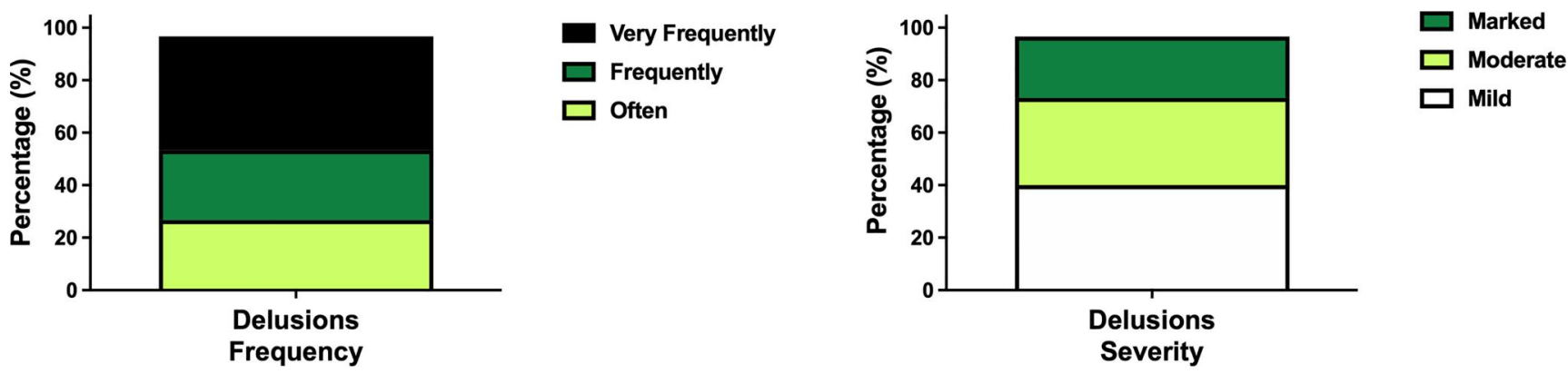

FIGURE 1 Frequency and severity of delusions as determined by the Neuropsychiatric Inventory (NPI)

on a one-to-one basis. The matching was conducted hierarchically for the following variables: (i) diagnosis and certainty, (ii) clinical disease stage (FRS), (iii) sex, (iv) age and (v) education. All cases were matched on at least the first two variables and were selected blind to genetic mutation status.

\subsubsection{Genetic and family history screening}

Because this is not a formal genetic study, genetic screening is conducted based on several factors (e.g., family history, clinical diagnosis). The number of individuals in the subgroup analysis with available genetic data is shown in Table 3. In the Delusions+ group, $11 / 27$ individuals had a positive gene mutation compared to only $1 /$ 26 in the Delusions- group (8\%). In the Delusions+ groups, the most common gene mutation was C9orf72 (8/27 screened), whereas none of the 26 Delusions-patients which were screened for C9orf72 had a positive result. The distribution of Goldman scores indicated a significantly higher number of people with a strong family history in the Delusions+ group ${ }^{\dagger}\left(x^{2}=5.994, p=0.014\right)$, with 13 people in the Delusions+ group and 5 people in Delusions- group reporting a strong family history (Goldman score <3.5).

\subsection{2 | Behavioural and cognitive profile}

The Delusions+ group had more abnormal beliefs reported on the CBI than the Delusions- group (Table 4, $p<0.001$ ). Notably, the
Delusions+ group also had greater impairments in everyday skills ( $p=0.004)$, stereotypical behaviours $(p=0.031)$, sleep disturbances $(p=0.003)$, memory problems $(p=0.012)$ and mood disturbances $(p=0.017)$. On the NPI, the Delusions+ group also had more hallucinations than the Delusions- group $(p=0.006)$ and a trend for greater disinhibition $(p=0.078)$ and anxiety ( $p=0.076$ ) (Table 4).

Examination of the cognitive profile of the Delusions+ and Delusions- groups revealed more severe cognitive deficits in the Delusions+ group (Supplementary Table 2). Specifically, the Delusions + group performed worse than the Delusions- group on the ACE ( $p=0.035)$, in particular, the attention/orientation $(p=0.022)$ and memory ( $p=0.013$ ) subtests. In addition, the Delusions+ group had worse visuospatial memory than the Delusions- group (Rey Complex Figure copy; $p=0.006$ ) and slightly reduced capacity to repeat words on the SYDBAT (Delusions+ vs. controls, $p=0.001$; Delusions+ vs. Delusions-, $p=0.040$ ) (See Supplementary Table 2).

\subsection{3 | Neuroimaging analyses}

In the Delusions+ group, more severe delusional psychopathology was correlated with decreased grey matter integrity in the right middle frontal gyrus and right temporoparietal junction, encroaching into the planum temporale and the supramarginal gyrus, as well as the left anterior temporal lobe, left temporal pole and left middle and inferior temporal gyrus (see Figure 2 and Supplementary Table 1). 


\begin{tabular}{lll} 
\% With genetic screening & Delusions $+n=27 / 30(90 \%)$ & Delusions- $n=26 / 30(87 \%)$ \\
\hline C9orf72 & $8 / 27(30 \%)$ & $0 / 26(0 \%)$ \\
GRN & $2 / 13(15 \%)$ & $1 / 14(7 \%)$ \\
\hline MAPT & $1 / 14(7 \%)$ & $0 / 15(0 \%)$
\end{tabular}

Note: Numbers refer to: number of individuals tested positive/number of individuals tested for the gene abnormality.

Abbreviations: GRN, granulin precursor; MAPT, microtubule associated protein tau.

\begin{tabular}{|c|c|c|c|c|}
\hline & Delusions+ & Delusions- & $t$ statistic & $p$ \\
\hline \multicolumn{5}{|l|}{$\mathrm{CBI}$} \\
\hline Memory & $62.89 \pm 19.31$ & $49.14 \pm 21.91$ & 2.58 & 0.012 \\
\hline Everyday skills & $56.54 \pm 31.57$ & $33.88 \pm 26.00$ & 3.04 & 0.004 \\
\hline Self-care & $25.76 \pm 27.44$ & $15.42 \pm 21.51$ & 1.63 & 0.110 \\
\hline Mood & $43.13 \pm 20.98$ & $29.58 \pm 21.71$ & 2.46 & 0.017 \\
\hline Beliefs & $33.89 \pm 25.89$ & $3.89 \pm 7.17$ & 6.12 & $<0.001$ \\
\hline Abnormal behaviour & $40.14 \pm 23.25$ & $31.69 \pm 22.25$ & 1.44 & 0.155 \\
\hline Eating habits & $44.17 \pm 30.26$ & $32.43 \pm 25.33$ & 1.63 & 0.109 \\
\hline Sleep & $57.92 \pm 34.35$ & $32.92 \pm 26.97$ & 3.14 & 0.003 \\
\hline Stereotypical behaviour & $56.25 \pm 33.72$ & $39.10 \pm 25.85$ & 2.21 & 0.031 \\
\hline Motivation & $67.17 \pm 31.91$ & $61.17 \pm 32.18$ & 0.73 & 0.471 \\
\hline \multicolumn{5}{|l|}{ NPI } \\
\hline Agitation & $3.03 \pm 2.99$ & $2.13 \pm 2.69$ & 1.23 & 0.225 \\
\hline Anxiety & $2.93 \pm 3.70$ & $1.43 \pm 2.62$ & 1.81 & 0.076 \\
\hline Apathy & $5.33 \pm 3.55$ & $4.30 \pm 3.86$ & 1.08 & 0.285 \\
\hline Appetite & $4.37 \pm 4.17$ & $4.70 \pm 3.90$ & 0.32 & 0.750 \\
\hline Depression & $2.00 \pm 3.30$ & $1.07 \pm 2.4$ & 1.24 & 0.222 \\
\hline Disinhibition & $3.97 \pm 4.71$ & $2.23 \pm 2.36$ & 1.80 & 0.078 \\
\hline Elation & $1.00 \pm 2.59$ & $0.70 \pm 1.75$ & 0.53 & 0.600 \\
\hline Hallucinations & $1.20 \pm 2.22$ & $0.00 \pm 0.00$ & 2.96 & 0.006 \\
\hline Irritability & $3.00 \pm 3.95$ & $1.67 \pm 2.60$ & 1.54 & 0.129 \\
\hline Sleep & $2.37 \pm 3.39$ & $1.70 \pm 3.63$ & 0.74 & 0.465 \\
\hline Aberrant motor behaviour & $4.27 \pm 4.18$ & $2.60 \pm 3.57$ & 1.66 & 0.102 \\
\hline
\end{tabular}

Note: Values are mean \pm standard deviation.

Abbreviations: $\mathrm{CBI}$, Cambridge Behavioural Inventory; NPI, neuropsychiatric inventory composite score (i.e., frequency $\times$ severity).

\section{4 | DISCUSSION}

Here, we examined the presence, nature and neural correlates of delusions in a consecutive cohort of patients presenting to the Frontier dementia clinic in Sydney. Approximately $10 \%$ of the $>450$ patients experienced delusions at presentation, with delusions more common in patients diagnosed with frontotemporal dementia (bvFTD and FTD-MND) and Alzheimer's disease (AD). Patients with delusions
TABLE 3 Findings from genetic screening in the Delusions+ and Delusions- groups
TABLE 4 Ratings of behaviour and neuropsychiatric features in patients with and without delusions 


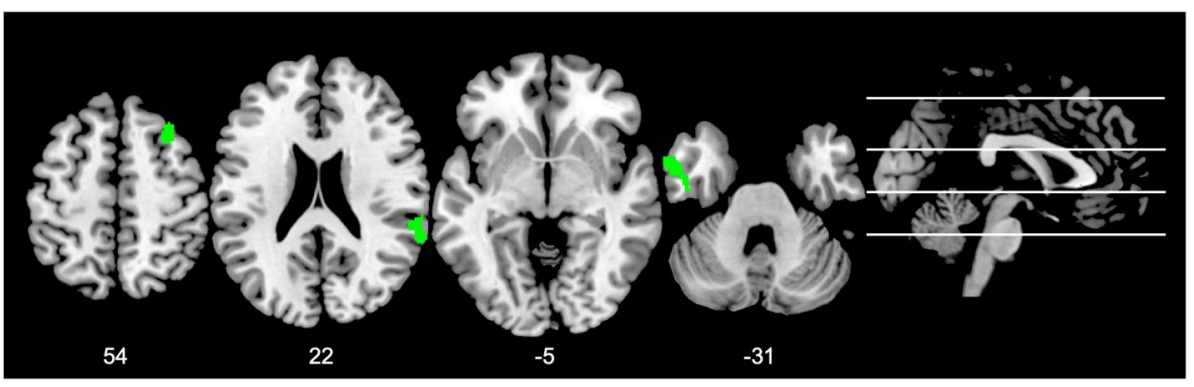

FIGURE 2 Regions that correlated with increased delusional psychopathology (NPI composite score) within the Delusions+ group. Left side of the brain is shown on the left side of the figure. Diagnosis group, age at scan, sex and disease severity (FRS Rasch Score) were included as covariates. Analyses are reported at $p<0.005$ voxelwise, uncorrected for multiple comparisons with a minimum cluster size of 100 voxels

diagnosed with primary progressive aphasia (SD, PNFA, LPA) or a motor disorder (CBS, PSP), rarely experienced delusions. A recent meta-analysis reported delusions in $\sim 30 \%$ of drug-naïve $A D$ patients. $^{35}$ This higher prevalence may reflect differences in disease stage, whereby delusions may increase with disease severity. ${ }^{35}$ Here, we focused on $A D$ patients at first presentation, which concurs with previous findings of a prevalence of $8 \%-16 \%$ in the very mild-mild stages of $A D .^{35}$ In bvFTD, previously reported rates of delusions are highly variable, ranging from $2.3 \%^{5}$ to as high as $17 \%-20 \%{ }^{36,37}$ Our results indicate that the upper end of this scale is more likely to be accurate, with $18.4 \%$ of bvFTD patients experiencing delusions at first presentation. The high presence of delusions likely contributes to the $\sim 50 \%$ of bvFTD patients who are initially diagnosed with a primary psychiatric disorder. ${ }^{3,38}$ Interestingly some evidence suggests that patients clinically diagnosed with FTD are more likely to have Alzheimer's pathology if their clinical profile included delusions. ${ }^{37}$ While pathological data are not currently available in this cohort, it will be important to follow these bvFTD patients to determine the proportion of these clinically diagnosed patients who are actually harbouring Alzheimer's pathology.

A second important variable, which may account for the relatively high proportion of patients diagnosed with both bvFTD and FTD-MND with delusions, are genetic abnormalities and/or a strong family history of disease. ${ }^{39}$ Psychotic symptoms, including delusions, have been reported to be substantially more common in patients carrying the C9orf72 gene expansion, than those without. ${ }^{40}$ Here, we found evidence that abnormalities in the three major FTD genes, C9orf72, MAPT and GRN, were present in people with delusions. Indeed, patients with delusions were more likely to have a C9orf72 expansion, which aligns with previous findings in the field. ${ }^{36}$ Our results highlight that in individuals who are presenting with novel neuropsychiatric symptoms, later in life, differential diagnosis should include neurodegenerative as well as psychiatric aetiologies. ${ }^{16}$ Future studies are essential to elucidate biomarkers that can more effectively differentiate between psychiatric and neurodegenerative disorders. $^{38}$

A number of cognitive neuropsychiatric theories of delusions have been proposed to account for the emergence of monothematic delusions. ${ }^{41,42}$ The two-factor model of delusions, suggests that delusions arise from: (i) a neuropsychological impairment which invokes an abnormal experience and explains the specific content of the individual's delusion and (ii) impaired belief reasoning, such that the delusional belief is irrationally adopted and maintained in the face of abundant counter-evidence. ${ }^{42}$ Delusional content in this cohort was variable but notably most individuals with delusions reported more than one delusional theme. The predominant theme was persecutory, followed by delusions of reference and delusions of jealousy. In schizophrenia, persecutory delusions often co-occur with delusions of reference. ${ }^{43}$ Previous studies have reported that persecutory delusions are especially common in dementia, ${ }^{4}$ which accords with our findings.

Our regression model as well as comprehensive neuropsychological assessment confirmed that disease severity is an important predictor of delusions, which aligns with previous studies in Alzheimer's disease. ${ }^{44}$ In addition, the regression model found that cognitive impairment was also an important contributor to delusions. Between groups, the Delusions+ patients were more disoriented and had worse episodic memory. They also experienced more hallucinations, which may have triggered delusions in some cases, as is seen in psychosis. The presence of memory impairment may accord with predominantly persecutory delusions (e.g., belief that people are stealing things, people taking their money) or even polythematic delusions when different episodes of memory failure prompt different delusional explanations (e.g., delusional jealousy of a spouse). According to the two-factor model, the second factor, also required for the development and persistence of delusions, is deficient belief evaluation or reasoning capacity, ${ }^{45}$ purported to be associated with frontal dysfunction. ${ }^{46}$ Somewhat surprisingly, the Delusions+ group were not disproportionately impaired on any measure of executive functioning, although our neuropsychological battery was not specifically designed to comprehensively target executive functioning and reasoning. It was beyond the scope of this study to examine the specific profiles of cognitive impairment and determine how they relate to individual delusional content, or to compare the cognitive profiles of those with monothematic versus polythematic delusions; however, this warrants future investigation.

From a neurobiological perspective, our results extend previous research providing new insights into the emergence of delusions in brain disorders. Delusional psychopathology was associated with reduced integrity in the right middle frontal gyrus, left anterior 
temporal lobe and right supramarginal gyrus, which concords with recent lesion mapping studies of monothematic delusions and functional imaging studies of psychosis ${ }^{47}$ and may reflect its role in prediction error processing. ${ }^{48}$ A common neurobiological basis for delusions across neurodegenerative and psychiatric syndromes has been previously hypothesised, ${ }^{11}$ and is supported by the current results. The involvement of the right frontal cortex in the pathogenesis of delusions has been suggested by Coltheart ${ }^{46}$ and demonstrated by recent lesion mapping studies of monothematic delusions and functional imaging studies of psychosis. ${ }^{47,49}$ This brain region is also important in aspects of prediction error processing. ${ }^{48}$ While our neuroimaging analyses controlled for diagnosis, they cannot rule out the possibility that disease-specific neurocognitive mechanisms also contribute to the emergence of delusions. In Alzheimer's disease, impaired visuoperceptual function appears to be particularly important in the manifestation of misidentification type delusions, which may reflect atrophy in temporo-occipital brain regions, ${ }^{50}$ while persecutory delusions in Alzheimer's disease have been linked with frontostriatal dysfunction. Future studies with larger numbers of people with varying delusional subtype and dementia subtype, combined with neuroimaging data will be invaluable in examining these relationships further.

In the context of neurodegenerative disorders, delusions and hallucinations are increasingly recognised as important clinical phenomena in Dementia with Lewy Bodies and Parkinson's Disease Dementia. $^{2}$ In these syndromes, a spectrum of psychosis has been proposed, with symptoms ranging from minor illusions, passage hallucinations and presence hallucinations, through to delusions and multimodal hallucinations at the more severe end of the spectrum. Importantly, these symptoms have been linked to distinct neurobiological mechanisms. Delusions and loss of insight have been linked with widespread cortical Lewy Body pathology. Our results converge and suggest that atrophy across multiple brain regions underpin the manifestation of delusions, which is associated with greater cognitive impairment and disease stage. While individuals with Parkinson's disease Dementia and Dementia with Lewy Bodies were not available for testing as part of this cohort, comparison of these syndromes is an interesting avenue for future work. Moreover, investigation of clinicopathological relationships will be important for future studies, to understand the relationship between the nature of delusions and the location and type of pathological features.

Clinically, delusions can be very distressing. From a management perspective, recent evidence has shown that the presence of delusions is a predictor of institutionalisation. ${ }^{51}$ Furthermore, worryingly, the use of antipsychotic medication is a predictor of mortality. ${ }^{51}$ How can our results inform improved clinical management? First, our results demonstrate that routine screening of delusions is an important aspect of clinical assessment, particularly in bvFTD, FTD-MND and AD, and individuals with genetic abnormalities and/or a strong family history of disease. Second, our results suggest that delusions are more likely to be present in individuals with greater cognitive impairment. The need for treatment of delusions may depend on the delusional content. Where delusions are relatively benign, it may be more appropriate to educate carers about the cognitive basis of delusions and advise them that rationalisation of delusional beliefs is unlikely to be effective. Thus, environmental modification, informed by the delusional belief and nature of cognitive impairment may be an appropriate management strategy.

\section{ACKNOWLEDGEMENTS}

The authors are grateful to all the patients and their families for supporting our research. This work was supported in part by funding to ForeFront, a collaborative research group dedicated to the study of frontotemporal dementia and motor neuron disease, from the National Health and Medical Research Council (NHMRC) (GNT1037746, GNT1095127) and the Australian Research Council (ARC) Centre of Excellence in Cognition and its Disorders Memory Program (CE11000102), including a Cross Program Support Scheme grant. Fiona Kumfor is supported by an NHMRC Career Development Fellowship (GNT1158762). Jessica L. Hazelton is supported by an NHMRC Postgraduate Scholarship (GNT1168597). Olivier Piguet is supported by an NHMRC Senior Research Fellowship (GNT1103258). John B. Kwok is supported by an NHMRC Dementia Research Team Grant (GNT1095127). Carol Dobson-Stone is supported by an NHMRC Boosting Dementia Research Leadership Fellowship (GNT1138223). Glenda M. Halliday is a NHMRC Senior Principal Research Fellow (GNT1079679). Ramon Landin-Romero is supported by the Appenzeller Neuroscience Fellowship in Alzheimer's Disease and the ARC Centre of Excellence in Cognition and its Disorders Memory Program (CE110001021). The authors acknowledge the Sydney Informatics Hub at the University of Sydney for providing access to High Performance Computing resources. Open access publishing facilitated by The University of Sydney, as part of the Wiley - The University of Sydney agreement via the Council of Australian University Librarians.

\section{CONFLICT OF INTEREST}

The authors have no Conflicts of Interest to declare.

\section{ETHICS APPROVAL}

This study was performed in line with the principles of the Declaration of Helsinki. Approval was granted by the South Eastern Sydney Local Health District Human Research Ethics Committee (HREC ref no. $10 / 126 \& 13 / 177)$.

\section{CONSENT TO PARTICIPATE}

Informed consent was obtained from all individual participants included in the study.

\section{CONSENT FOR PUBLICATION}

Patients provided informed consent regarding publishing their data.

\section{AUTHOR CONTRIBUTIONS}

Conceptualisation: Fiona Kumfor, Emma Devenney, Emily Connaughton, Robyn Langdon, Ramon Landin-Romero; Methodology: Fiona Kumfor, Cheng Tao Liang, Jessica L. Hazelton, Cristian E. 
Leyton, Emily Connaughton, Robyn Langdon, Eneida Mioshi, John B. Kwok, Carol Dobson-Stone, Olivier Piguet, Ramon Landin-Romero; Data curation: Fiona Kumfor, Cheng Tao Liang, Jessica L. Hazelton, Cristian E. Leyton, Cassandra Kaizik, Emma Devenney, Emily Connaughton, Robyn Langdon, Eneida Mioshi, John B. Kwok, Carol Dobson-Stone, Glenda M. Halliday, John R. Hodges, Ramon LandinRomero; Formal Analysis and investigation: Fiona Kumfor, Cheng Tao Liang, Jessica L. Hazelton, Cristian E. Leyton, Cassandra Kaizik, Emma Devenney, Emily Connaughton, Robyn Langdon, Eneida Mioshi, John B. Kwok, Carol Dobson-Stone, Glenda M. Halliday, Olivier Piguet, John R. Hodges, Ramon Landin-Romero; Visualisation: Fiona Kumfor, Cheng Tao Liang, Jessica L. Hazelton, Cristian E. Leyton, Ramon Landin-Romero; Writing - original draft: Fiona Kumfor, Ramon Landin-Romero; Writing - review and editing: Fiona Kumfor, Emily Connaughton, Robyn Langdon, Eneida Mioshi, Glenda M. Halliday, Olivier Piguet, John R. Hodges, Ramon Landin-Romero; Validation: Fiona Kumfor, Glenda M. Halliday, Olivier Piguet, John R. Hodges, Ramon Landin-Romero; Project administration: Fiona Kumfor, John R. Hodges, Ramon Landin-Romero; Funding acquisition: Fiona Kumfor, Glenda M. Halliday, Olivier Piguet, John R. Hodges, Ramon Landin-Romero; Resources: Glenda M. Halliday, Olivier Piguet, John R. Hodges; Supervision: Olivier Piguet, John R. Hodges.

\section{DATA AVAILABILITY STATEMENT}

The data generated during, or analysed as part of the current study, are available from the corresponding author on reasonable request.

\section{ORCID}

Fiona Kumfor (iD https://orcid.org/0000-0002-3208-075X

\section{ENDNOTES}

* Data were missing for the frequency and severity of symptoms in one Delusions+ participant whose informant had answered 'yes' to the delusions screening question. To take a conservative approach for our analyses, this person was nevertheless included in the Delusions+ group.

** Includes 1 patient diagnosed with posterior cortical atrophy.

${ }^{\dagger}$ Goldman scores not available for 2 people in the Delusions+ group.

\section{REFERENCES}

1. American Psychiatric Association. Diagnostic and Statistical Manual of Mental Disorders (DSM-5®). American Psychiatric Pub; 2013.

2. Creese B, Politis M, Chaudhuri KR, Weintraub D, Ballard C, Aarsland D. The psychosis spectrum in Parkinson disease. Nat Rev Neurol. 2017;13(2):81-95.

3. Woolley JD, Khan BK, Murthy NK, Miller BL, Rankin KP. The diagnostic challenge of psychiatric symptoms in neurodegenerative disease; rates of and risk factors for prior psychiatric diagnosis in patients with early neurodegenerative disease. J Clin Psychiatry. 2011;72(2):126-133.

4. Bassiony MM, Lyketsos CG. Delusions and hallucinations in Alzheimer's disease: review of the brain decade. Psychosomatics. 2003; 44(5):388-401.
5. Mendez MF, Shapira JS, Woods RJ, Licht EA, Saul RE. Psychotic symptoms in frontotemporal dementia: prevalence and review. Dementia Geriatr Cognit Disord. 2008;25(3):206-211.

6. Lillo P, Garcin B, Hornberger M, Bak TH, Hodges JR. Neurobehavioral features in frontotemporal dementia with amyotrophic lateral sclerosis. Arch Neurol. 2010;67(7):826-830.

7. Harciarek $M$, Kertesz $A$. The prevalence of misidentification syndromes in neurodegenerative diseases. Alzheimer Dis Assoc Disord. 2008;22(2):163-169.

8. Insel T, Cuthbert B, Garvey M, et al. Research Domain criteria (RDoC): toward a new classification framework for research on mental disorders. Am J Psychiatry. 2010;167(7):748-751.

9. Doucet GE, Janiri D, Howard R, O'Brien M, Andrews-Hanna JR, Frangou S. Transdiagnostic and disease-specific abnormalities in the default-mode network hubs in psychiatric disorders: a meta-analysis of resting-state functional imaging studies. Eur Psychiatry. 2020; 63(1):e57.

10. Ahmed RM, Bocchetta M, Todd EG, et al. Tackling clinical heterogeneity across the amyotrophic lateral sclerosis-frontotemporal dementia spectrum using a transdiagnostic approach. Brain Commun 2021;3(4):fcab257.

11. Reeves SJ, Gould RL, Powell JF, Howard RJ. Origins of delusions in Alzheimer's disease. Neurosci Biobehav Rev. 2012;36(10):2274-2287.

12. Gorno-Tempini ML, Hillis AE, Weintraub S, et al. Classification of primary progressive aphasia and its variants. Neurology. 2011; 76(11):1006-1014.

13. Höglinger GU, Respondek G, Stamelou M, et al. Clinical diagnosis of progressive supranuclear palsy: the movement disorder society criteria. Mov Disord. 2017;32(6):853-864.

14. Mathew R, Bak TH, Hodges JR. Diagnostic criteria for corticobasal syndrome: a comparative study. J Neurol Neurosurg Psychiatry. 2012;83(4):405-410.

15. McKhann GM, Knopman DS, Chertkow $\mathrm{H}$, et al. The diagnosis of dementia due to Alzheimer's disease: recommendations from the National Institute on Aging-Alzheimer's Association workgroups on diagnostic guidelines for Alzheimer's disease. Alzheimer's dementia. 2011;7(3):263-269.

16. Rascovsky K, Hodges JR, Knopman DS, et al. Sensitivity of revised diagnostic criteria for the behavioural variant of frontotemporal dementia. Brain. 2011;134(9):2456-2477.

17. Strong MJ, Grace GM, Freedman M, et al. Consensus criteria for the diagnosis of frontotemporal cognitive and behavioural syndromes in amyotrophic lateral sclerosis. Amyotroph Lateral Scler. 2009;10(3): 131-146.

18. So M, Foxe D, Kumfor F, et al. Addenbrooke's cognitive examination III: psychometric characteristics and relations to functional ability in dementia. J Int Neuropsychological Soc. 2018;24(8):854-863.

19. Cummings JL. The Neuropsychiatric Inventory: assessing psychopathology in dementia patients. Neurology. 1997;48(5 Suppl 6): 10S-16S.

20. Structured Clinical Interview for DSM-5 Research Version (SCID-5 for DSM-5, Research Version; SCID-5-RV). American Psychiatric Association; 2015.

21. Förstl H, Besthorn C, Burns A, Geiger-Kabisch C, Levy R, Sattel A. Delusional misidentification in Alzheimer's disease: a summary of clinical and biological aspects. Psychopathology. 1994;27(3-5): 194-199.

22. Goldman JS, Farmer JM, Wood EM, et al. Comparison of family histories in FTLD subtypes and related tauopathies. Neurology. 2005; 65(11):1817.

23. Dobson-Stone C, Hallupp M, Bartley L, et al. C9ORF72 repeat expansion in clinical and neuropathologic frontotemporal dementia cohorts. Neurology. 2012;79(10):995. 
24. Dobson-Stone C, Kwok JBJ. Finding MAPT mutations in frontotemporal dementia and other tauopathies. In: Smet-Nocca C, ed. Tau Protein: Methods and Protocols. Springer New York; 2017:307-324.

25. Schofield EC, Halliday GM, Kwok J, Loy C, Double KL, Hodges JR. Low serum progranulin predicts the presence of mutations: a prospective study. J Alzheimer's Dis. 2010;22(3):981-984.

26. Dobson-Stone C, Shaw AD, Hallupp M, et al. Is CHCHD10 Pro34Ser pathogenic for frontotemporal dementia and amyotrophic lateral sclerosis? Brain. 2015;138(10):e385.

27. Savage S, Hsieh S, Leslie F, Foxe D, Piguet O, Hodges JR. Distinguishing subtypes in primary progressive aphasia: application of the Sydney language battery. Dementia geriatric cognitive Disord. 2013;35(3-4):208-218.

28. Burgess P, Shallice T. The Hayling and Brixton Tests. Thames Valley Test Company Bury St. Edmonds; 1997.

29. Wear HJ, Wedderburn CJ, Mioshi E, et al. The Cambridge behavioural inventory revised. Dementia Neuropsychologia. 2008;2(2): 102-107.

30. Smith SM, Jenkinson M, Woolrich MW, et al. Advances in functional and structural MR image analysis and implementation as FSL. Neuroimage. 2004;23(Suppl 1):S208-S219.

31. Nichols TE, Holmes AP. Nonparametric permutation tests for functional neruoimaging: a primer with examples. Hum Brain Mapp. 2001;15:1-25

32. Mioshi E, Hsieh S, Savage S, Hornberger M, Hodges JR. Clinical staging and disease progression in frontotemporal dementia. Neurology. 2010;74(20):1591-1597.

33. Lieberman MD, Cunningham WA. Type I and Type II error concerns in fMRI research: re-balancing the scale. Soc Cognitive Affect Neurosci. 2009;4(4):423-428.

34. Elm E, Altman D, Egger M, Pocock S, Gotzsche P, Vandenbroucke J. Strengthening the reporting of observational studies in epidemiology (STROBE) statement: guidelines for reporting observational studies. BMJ. 2007;335:806-808.

35. Lai L, Lee PE, Chan P, Fok MC, Hsiung G-YR, Sepehry AA. Prevalence of delusions in drug-naïve Alzheimer disease patients: a metaanalysis. Int J Geriatric Psychiatry. 2019;34(9):1287-1293.

36. Hall D, Finger EC. Psychotic symptoms in frontotemporal dementia. Curr Neurology Neurosci Rep. 2015;15(7):46.

37. Léger GC, Banks SJ. Neuropsychiatric symptom profile differs based on pathology in patients with clinically diagnosed behavioral variant frontotemporal dementia. Dementia Geriatric Cognitive Disord. 2014; 37(1-2):104-112.

38. Ducharme S, Dols A, Laforce R, et al. Recommendations to distinguish behavioural variant frontotemporal dementia from psychiatric disorders. Brain. 2020;143(6):1632-1650.

39. Varghese D, Wray NR, Scott JG, Williams GM, Najman JM, McGrath JJ. The heritability of delusional-like experiences. Acta Psychiatr Scand. 2013;127(1):48-52.

40. Devenney EM, Landin-Romero R, Irish M, et al. The neural correlates and clinical characteristics of psychosis in the frontotemporal dementia continuum and the C9orf72 expansion. Neurolmage Clinical. 2017;13:439-445.

41. Halligan PW, David AS. Cognitive neuropsychiatry: towards a scientific psychopathology. Nat Rev Neurosci. 2001;2(3):209.

42. Coltheart M, Langdon R, McKay R. Delusional belief. Annu Rev Psychol. 2011;62:271-298.

43. Sartorius N, Jablensky A, Korten A, et al. Early manifestations and first-contact incidence of schizophrenia in different cultures: a preliminary report on the initial evaluation phase of the WHO Collaborative Study on Determinants of Outcome of Severe Mental Disorders. Psychol Med. 1986;16(4):909-928.

44. Hashimoto $M$, Yatabe $Y$, Ishikawa $T$, et al. Relationship between dementia severity and behavioral and psychological symptoms of dementia in dementia with Lewy Bodies and Alzheimer's disease patients. Dement Geriatr Cogn Dis Extra. 2015;5(2):244-252.

45. Coltheart M, Menzies P, Sutton J. Abductive inference and delusional belief. Cogn Neuropsychiatry. 2010;15(1-3):261-287.

46. Coltheart M. Cognitive neuropsychiatry and delusional belief. Q J Exp Psychol. 2007;60(8):1041-1062.

47. Darby RR, Laganiere S, Pascual-Leone A, Prasad S, Fox MD. Finding the imposter: brain connectivity of lesions causing delusional misidentifications. Brain. 2017;140(2):497-507.

48. Corlett PR, Fletcher PC. Delusions and prediction error: clarifying the roles of behavioural and brain responses. Cogn Neuropsychiatry. 2015;20(2):95-105.

49. Devinsky O. Delusional misidentifications and duplications. Neurology. 2009;72(1):80.

50. Reeves SJ, Clark-Papasavas C, Gould RL, Ffytche D, Howard RJ. Cognitive phenotype of psychotic symptoms in Alzheimer's disease: evidence for impaired visuoperceptual function in the misidentification subtype. Int J Geriatr Psychiatry. 2015;30(12):1147-1155.

51. Connors MH, Ames D, Woodward M, Brodaty H. Psychosis and clinical outcomes in Alzheimer disease: a longitudinal study. Am J Geriatric Psychiatry. 2018;26(3):304-313.

\section{SUPPORTING INFORMATION}

Additional supporting information may be found in the online version of the article at the publisher's website.

How to cite this article: Kumfor F, Liang CT, Hazelton JL, et al. Examining the presence and nature of delusions in Alzheimer's disease and frontotemporal dementia syndromes. Int J Geriatr Psychiatry. 2022;1-10. https://doi.org/10.1002/ gps.5692 